\title{
Cognitive behavioural therapy has short term but not long term benefits in people with residual symptoms of depression
}

Paykel ES, Scott J, Cornwall PL, et al. Duration of relapse prevention after cognitive therapy in residual depression: follow-up of controlled trial. Psychological Med 2005;35:59-68.

Does cognitive behavioural therapy reduce the risk of relapse in people with residual symptoms of depression?

\section{METHODS}

Design: Retrospective follow up of an earlier randomised
controlled trial.

\section{MAIN RESULTS}

In a previously reported phase of this prospective randomised controlled trial, CBT plus clinical management reduced relapse rates at 20 weeks and 16 months. In this study, participants were reinterviewed a mean of six years after randomisation. There were no significant differences in relapse rates between CBT plus clinical management and clinical management alone at any stage between 16 months and 6 years (relapse rates: at about 2.5 years, 38\% with CBT plus clinical management $v 43 \%$ with clinical management alone, $\mathrm{p}=0.25$; at about 6 years: $60 \%$ with $\mathrm{CBT}$ plus clinical management $v 65 \%$ with clinical management alone, $\mathrm{p}=0.33$ ). Similarly, differences in severity of depression were also only apparent in the first two years of follow up.

For correspondence: Professor E S Paykel, Department of Pyschiatry, University of Cambridge, Douglas House, 18E Trumpington Road, Cambridge CB2 2AH, UK

Sources of funding: none declared.

\section{CONCLUSIONS}

Cognitive behavioural therapy plus clinical management reduces the short term but not the long term risk of relapse compared with clinical management alone, in people with residual symptoms of depression.

\section{NOTES}

In the long term retrospective phase of the study, participants were expected to recall information from the previous 4.5 years to help ascertain if relapse had occurred. Thus, even though some of this information was obtained from hospital records, relapse data may have been influenced by the participant's ability to recall information over a long time period.

\section{Commentary}

M ajor depressive disorder is often highly recurrent, and prevention of recurrence has generally received less study than acute treatment. Still, a number of trials have shown that cognitive behavioral therapy (CBT) aimed at residual depressive symptoms is effective in reducing risk of recurrence.

In the recent report by Paykel et al, the benefit of CBT persisted for more than three years after CBT was terminated. However, beyond about three years the benefit of CBT appears to decay. This result differs from the findings of another long term follow up study, by Fava et al. ${ }^{1}$

The two studies differ in several ways, most notably in the use of pharmacotherapy. While Fava et al tapered antidepressant treatment following remission, Paykel et al allowed it to be continued-about $60 \%$ of subjects in both arms were on medication at follow up. In addition, participants in Fava et al's study had relatively few residual depressive symptoms by design, while in Paykel et al's study participants had substantial residual symptoms $(\mathrm{HAMD} \geqslant 8)$. That both studies observed similar benefit over the first several years, despite these differences, suggests that the use of CBT following an acute episode may be useful in a broad range of people with major depressive disorder. On the other hand, the subsequent discordance may provide further support for the negative prognostic value of residual symptoms. ${ }^{2}$

CBT remains costly (as does pharmacotherapy) and it is not always readily accessible in all areas. Therefore, while its efficacy has been demonstrated convincingly, clinicians also need information about when CBT should be applied, particularly over the long term. Paykel et al's result suggests that the underlying vulnerability to depression remains even in those people who maintain a response to CBT over several years. Further intervention may be required for some people with major depressive disorder.

It is tempting to speculate about the value of "refresher" or "tune up" $\mathrm{CBT}$, perhaps after the re-emergence of depressive symptoms. For now, this fits with a general principle of good practice: people who have suffered a depressive episode require long term follow up, with close attention to the re-emergence of depressive symptoms. The study by Paykel et al reminds clinicians that recurrent major depression is a disorder that is effectively treated, but not necessarily cured, by CBT. Roy H Perlis, MD Massachusetts General Hospital and Harvard Medical School, Boston, MA, USA

1 Fava GA, Ruini C, Rafanelli C, et al. Six-year outcome of cognitive behavior therapy for prevention of recurrent depression. Am J Psychiatry 2004;161:1872-6

2 Boulenger JP. Residual symptoms of depression: clinical and theoretical implications. Eur Psychiatry 2004;19:209-13. 